\title{
SOLUCIÓN DE UN PROBLEMA COTIDIANO MEDIANTE UN ANÁLISIS TERMODINÁMICO
}

\author{
Miquel Fornells Gallart \\ Catedrático del Instituto de Enseñanza Secundaria Les Corts de Barcelona.
}

Recibido 22-X-2004

Aceptado 8-II-2005

\section{Introducción}

Las Ciencias de la Naturaleza como ciencias experimentales que son, desde el punto de vista del sistema constructivista, necesitan un aprendizaje significativo, es decir, dar prioridad: a aprendizajes que

Resumen: Cuando un globo de feria se escapa libremente a la atmósfera queda sometido a una serie de factores físicos y químicos que al final van a limitar la altura máxima que alcanzará. En un primer paso y utilizando el principio de Arquímedes se calcula la densidad del aire en el punto de máxima altura. A continuación, suponiendo un modelo adiabático de la atmósfera, se encuentra una expresión que relaciona las densidades del aire en la superficie terrestre y en el punto de altura máxima con las presiones en dichos puntos. Finalmente la ecuación fundamental de la hidrostática nos permite obtener el valor numérico de dicha altura máxima, cosa que es el objetivo del trabajo. Por otra parte se hace un estudio de la influencia que puede tener durante el trayecto de elevación del globo factores como el cambio de temperatura y el cambio de presión, demostrándose que no son factores que puedan tener una incidencia significativa en el resultado obtenido.

Palabras clave: Constructivismo, adiabático, fluido, diatómico, gradiente, entalpía libre. se realicen a través de la experiencia, a la comprensión razonada de lo que se hace y a los procedimientos que se utilizan para resolver las actividades.

Así, en esta metodología constructivista, los alumnos son constructores de su propio aprendizaje. Para ello hay muchas
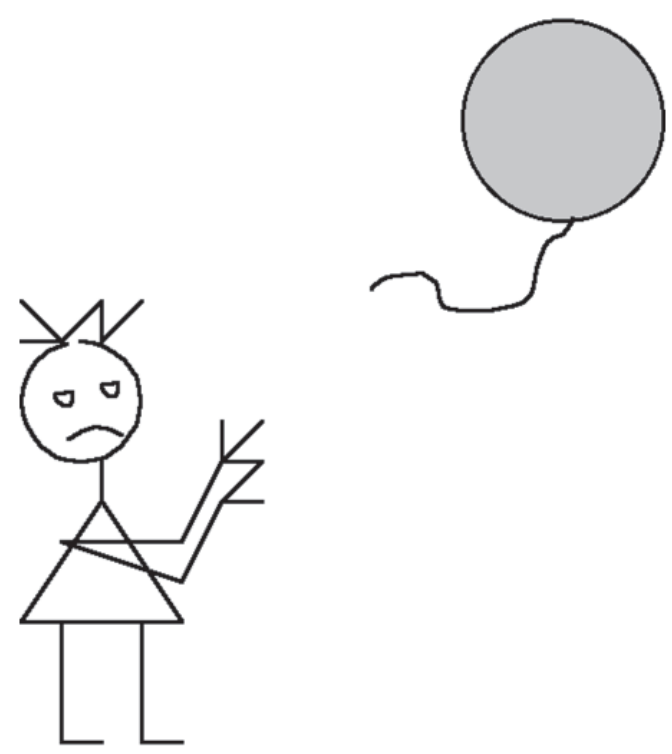


\begin{abstract}
When a fairground balloon flies up the sky, it remains subject to different physical and chemical elements which at the end will put an end to the maximum height that it will reach. First, and making use of Arquimedes's principle, one calculates the air density in the point of maximum height. Next, using an adiabatic model of the atmosphere, we find one expression which relates the ire densities in the surface of Earth and in the point of maximum height with the pressures in those points. At the end, the fundamental equation of the hydrostatic allows us to obtain the numerical value of the maximum height: the objective of this study. On the other hand, one studies the influence that the balloon can have during the route of elevation e.g. such factors as the change of pressure and temperature. It can be demonstrated that these factors do not have significant incidences in the results obtained.
\end{abstract}

Key words: Constructivism, adiabatic, fluid, diatomic, gradient, free energy. formas de actuar por parte del profesor para desarrollar una didáctica adecuada. Una de estas formas son, por ejemplo, los ejercicios propuestos para investigar fenómenos cotidianos muy comunes pero que no es tan sencillo explicar el porqué ocurren.

El ejemplo que se propone en este estudio es uno de estos fenómenos comúnmente observados como es la elevación de un globo de goma lleno de helio como los que se venden en las ferias para divertimento de niños.

Ha sido escogido este fenómeno puesto que abarca un amplio abanico de leyes físicas y, por tanto tienen el valor añadido que permite la comprensión, mediante la aplicación práctica, de una serie de principios de la física de los fluidos que van desde el principio de Arquímedes hasta el principio fundamental de la hidrostática pasando por diferentes ideas básicas de la termodinámica física y química.

Se trata pues de un fenómeno cotidiano cuyo estudio científico ayuda a comprender toda una serie de conocimientos de la física y de la química que demasiadas veces los alumnos conocen solamente desde un punto de vista teórico y lleno de abstracción. El estudio evidencia un claro componente didáctico para todos estos alumnos que se encuentran en la fase preuniversitaria o en los primeros cursos universitarios de ciencias e ingenierías.

\section{Descripción del sistema}

El sistema físico que se estudia está constituido por una parte de un globo de goma elástica de los típicos de feria o de espectáculos para fiestas. El globo está lleno con gas helio. Y por otra parte está constituido por la masa de aire atmosférico que envuelve al globo.

A veces se toman modelos isotermos de la atmósfera terrestre, en los cuales se considera que la temperatura no varía con la altura. Pero esto solamente es válido 
para alturas sobre la superficie terrestre comprendidas entre unos $10 \mathrm{~km}$ y unos 25 $\mathrm{km}$. Cuando la altura es inferior a $10 \mathrm{~km}$ existen movimientos continuos de masas de aire que suben y bajan. Cuando suben se expanden y se enfrían, y cuando bajan se comprimen y se calientan. Para representar este comportamiento se considera un modelo adiabático de la atmósfera en el cual se prescinde de la trasferencia de calor entre masas de aire adyacentes. Además se supone que el aire sigue un comportamiento ideal.

El objetivo del trabajo es determinar la altura a que subirá un globo teniendo presente este modelo y para ello, se tendrán en cuenta los siguientes datos:

- La densidad del gas que contiene el globo, el cual se supone que se trata de helio que es el normalmente utilizado en estos artilugios.

$$
\rho_{H e}=0,18 \frac{\mathrm{kg}}{\mathrm{m}^{3}}
$$

- $\quad$ El volumen del globo hinchado. Se puede considerar un globo esférico de radio: $R=15 \mathrm{~cm}$ y por tanto, suponiendo el globo aproximadamente esférico, corresponde a un volumen de:

$$
V=\frac{4}{3} \pi R^{3}=\frac{4}{3} \pi(0,15 m)^{3}=0,014 m^{3}
$$

- La masa del globo (sólo la goma que lo constituye) más la cuerda que lleva, es fácil de comprobar comprando uno de estos artilugios. Se determina un valor: $M=13 \mathrm{~g}=0,013 \mathrm{~kg}$.
- La densidad del aire más o menos a ras de suelo y a temperatura ambiente, que consultando en unas tablas resulta ser de:

$$
\rho_{0}=1,3 \frac{\mathrm{kg}}{\mathrm{m}^{3}}
$$

- La presión atmosférica normal a ras de suelo de:

$$
p_{0}=1 \mathrm{~atm}=1,013 \cdot 10^{5} \mathrm{~Pa}
$$

- La aceleración de la gravedad cerca de la superficie de la Tierra:

$$
g_{0}=9,81 \frac{m}{s^{2}}
$$

\section{Análisis del sistema}

Para analizar el sistema descrito, vamos a dividir el análisis en tres partes: en primer lugar hacemos las consideraciones mecánicas en cuanto a la fuerza ascensional que hace subir el globo, cosa que se utiliza para calcular la densidad del aire en el supuesto punto de altura máxima; en segundo lugar se plantea la ecuación fundamental de la hidrostática la cual se utilizará para, en tercer y último lugar, combinar con la expresión que relaciona presión y volumen en una transformación adiabática.

\section{- La fuerza ascensional}

Teniendo presente el Principio de Arquímedes: "todo cuerpo sumergido en un fluido experimenta un empuje hacia arriba igual al peso de fluido desalojado", es fácil calcular la fuerza ascensional $\left(F_{a}\right)$ que hace subir el globo la cual prácticamente es igual al empuje $(E)$ o peso del aire desalojado.

Afinando más, se le puede restar el peso del gas helio $\left(P_{H e}\right)$ que contiene el globo:

$$
F_{a}=E-P_{H e}=M_{\text {aire desalojado }} \cdot g_{0}-M_{H e} \cdot g_{0}=V_{a} \cdot \rho_{0} \cdot g_{0}-V_{a} \cdot \rho_{H e} \cdot g_{0}
$$

Donde $V_{a}$ representa el volumen del globo que en muy buena aproximación coincide con el volumen de gas helio que contiene. Reordenando la ecuación y sustituyendo valores numéricos resulta para la fuerza ascensional: 


$$
F_{a}=V_{a} \cdot g_{0} \cdot\left(\rho_{0}-\rho_{H e}\right)=0,014 \mathrm{~m}^{3} \cdot 9,8 \frac{\mathrm{m}}{\mathrm{s}^{2}} \cdot\left(1,3 \frac{\mathrm{kg}}{\mathrm{m}^{3}}-0,18 \frac{\mathrm{kg}}{\mathrm{m}^{3}}\right)=0,15 \mathrm{~N}
$$

No obstante, esta fuerza ascensional es variable a medida que el globo sube ya que la densidad del aire también varía y, concretamente sabemos que dicha densidad va disminuyendo. Se debería encontrar una relación entre la densidad y la altura para poder resolver el problema.

Pero antes mediante la misma ecuación con la que se ha calculado la fuerza ascensional se puede determinar el valor de la densidad del aire a la altura máxima que llegue el globo. Sólo hace falta tener presente que en el punto de altura máxima $\left(h_{\max }\right)$ dicha fuerza ascensional $\left(F_{a}\right)$ tiene que ser igual al peso total del globo en sí, es decir al peso de la goma más la cuerda que conforman este globo. Sea $P$ la densidad en $h_{\max }$, se tiene:

$$
F_{a}^{\prime}=E-P_{H e}=V_{a} \cdot \rho \cdot g_{0}-V_{a} \cdot \rho_{H e} \cdot g_{0}=V_{a} \cdot g_{0} \cdot\left(\rho-\rho_{H e}\right)
$$

E igualando esta fuerza al peso del globo: $M \cdot g_{0}$ :

$$
V_{a} \cdot g_{0} \cdot\left(\rho-\rho_{H e}\right)=M \cdot g_{0}
$$

Resulta para la densidad del aire en el punto de altura máxima:

$$
\rho=\frac{M}{V_{a}}+\rho_{H e}=\frac{0,013 \mathrm{~kg}}{0,014 \mathrm{~m}^{3}}+0,18 \frac{\mathrm{kg}}{\mathrm{m}^{3}}=1,11 \frac{\mathrm{kg}}{\mathrm{m}^{3}}
$$

- $\quad$ El principio general de la hidrostática.

El principio general de la hidrostática relaciona presión y altura.

La presión de una columna de fluido de altura $h$, mediante este principio, se calcula:

$$
p=\rho \cdot g \cdot h
$$

Con $P$ igual a la densidad del fluido y $g$ la aceleración de la gravedad del lugar.

Se supone que a la altura que puede llegar el globo, la aceleración de la gravedad será prácticamente igual al valor en la superficie terrestre $\left(g_{0}\right)$ y se puede formular la última ecuación en forma diferencial entre presión y altura:

$$
d p=-\rho \cdot g_{0} \cdot d h
$$

Donde $\rho$ es la densidad del aire a cualquier altura $h$ y el signo menos nos indica que la presión disminuye con la altura.

Así se tiene una relación entre la presión y la altura. Para poder integrar esta ecuación se debe encontrar una relación general entre la presión y la densidad del aire, y así obtener la relación entre densidad y la altura que podría resolver el problema.

- Relación presión-volumen en una transformación adiabática.

Una vez hecha la suposición que la atmósfera está en equilibrio adiabático, es decir, que la presión y el volumen de aire a ras de suelo estarán relacionadas con la presión y el volumen en la altura máxima mediante la expresión de un proceso a calor constante, o sea:

$$
p_{0} \cdot V_{0}^{\gamma}=p \cdot V^{\gamma}
$$


Donde $p_{0} \mathrm{y} V_{0}$ representan la presión y el volumen de aire desalojado a ras de suelo y $p$ y $V$ la presión y el volumen de aire desalojado a la altura máxima que alcance el globo: $h_{\max }$. Se supone que lo mejor para el aire es tomar $\gamma=1,4$ que es la relación entre calores específicos $(C p / C v)$ en un gas diatómico.

Sustituyendo en la última expresión los volúmenes por el cociente entre masa y densidad, se obtiene:

$p_{0}\left(\frac{M}{\rho_{0}}\right)^{\gamma}=p\left(\frac{M}{\rho}\right)^{\gamma} \quad \Rightarrow \quad p_{0} \cdot \rho^{\gamma}=p \cdot \rho_{0}^{\gamma}$

o bien:

$$
\rho=\rho_{0}\left(\frac{p}{p_{0}}\right)^{\frac{1}{\gamma}} \quad \Rightarrow \quad \frac{p}{p_{0}}=\left(\frac{\rho}{\rho_{0}}\right)^{\gamma}
$$

La primera expresión la sustituimos en la ecuación diferencial: $d p=-\rho \cdot g_{0} \cdot d h$

y se tiene:

$$
d h=-\frac{d p}{\rho g_{0}}=-\frac{1}{\rho_{0} g_{0}}\left(\frac{p_{0}}{p}\right)^{\frac{1}{\gamma}} d p
$$

pasando a integrar definidamente esta expresión entre $h=0$ y $h=h_{\max }$ valores a los que corresponde presiones de $p_{0}$ y $p$ respectivamente:

$$
\int_{0}^{h_{\max }} d h=-\int_{p_{0}}^{p} \frac{1}{\rho_{0} g_{0}}\left(\frac{p_{0}}{p}\right)^{\frac{1}{\gamma}} d p=-\frac{p_{o}^{1 / \gamma}}{\rho_{0} g_{0}} \int_{p_{0}}^{p} \frac{d p}{p^{1 / \gamma}}
$$

Resolviendo la integral se tiene:

$$
h_{\max }=\frac{\gamma}{\gamma-1} \frac{1}{\rho_{0} g_{0}} p_{0}^{1 / \gamma}\left(p_{0}^{\frac{\gamma-1}{\gamma}}-p^{\frac{\gamma-1}{\gamma}}\right)=\frac{\gamma}{\gamma-1} \frac{1}{\rho_{0} g_{0}} p_{0}^{1 / \gamma} \cdot p_{0}^{1-\frac{1}{\gamma}}\left(1-\left(\frac{p}{p_{0}}\right)^{\frac{1-\gamma}{\gamma}}\right)
$$

Por tanto:

$$
h_{\max }=\frac{\gamma}{\gamma-1} \frac{1}{\rho_{0} g_{0}} p_{0}\left(1-\left(\frac{p}{p_{0}}\right)^{\frac{1-\gamma}{\gamma}}\right)
$$

y sustituyendo $\frac{p}{p_{0}}=\left(\frac{\rho}{\rho_{0}}\right)^{\gamma}$ resulta:

$$
h_{\max }=\frac{\gamma}{\gamma-1} \frac{p_{0}}{\rho_{0} g_{0}}\left(1-\left(\frac{\rho}{\rho_{0}}\right)^{\gamma-1}\right)
$$

Esta es la expresión que da la altura máxima a la que llegará el globo, expresión en la cual figuran todos los datos de que se disponía inicialmente.

Sustituyendo los valores numéricos correspondientes se obtiene:

$$
h_{\max }=\frac{1,4}{1,4-1} \cdot \frac{1,013 \cdot 10^{5} \mathrm{~Pa}}{1,3 \frac{\mathrm{kg}}{\mathrm{m}^{3}} \cdot 9,81 \frac{\mathrm{m}}{\mathrm{s}^{2}}} \cdot\left(1-\left(\frac{1.11 \mathrm{~kg} / \mathrm{m}^{3}}{1,3 \mathrm{~kg} / \mathrm{m}^{3}}\right)^{1,4-1}\right)=1704 \mathrm{~m}
$$


Así la altura máxima a la que sube el globo según los cálculos considerados es de $\mathbf{1 7 0 4} \mathbf{~ m}$.

\section{Otra situación aplicable a nuestro sistema}

Otro tipo de situación dentro del formalismo de la termodinámica, que resulta aplicable a nuestro sistema, es el caso de determinar el gradiente de temperatura en la atmósfera terrestre hasta la altura de unos $10 \mathrm{~km}$ sobre la superficie. Será lo que se puede llamar gradiente adiabático de la atmósfera (Grad).

$$
G r a d=-\frac{d T}{d h}
$$

Hay que calcular esta magnitud y de esta forma se puede saber si la disminución de temperatura con la altura puede influir en la evolución del globo mientras sube. Hay que buscar pues, alguna expresión que relacione este gradiente con parámetros conocidos.

Considerando una atmósfera con comportamiento de gas ideal, en primer lugar, se puede establecer mediante la ecuación de Clapeyron ${ }^{1}$ escrita en la forma:

$$
\frac{d T}{T}=\frac{R}{c_{p}} \frac{d p}{p}
$$

Volviendo al principio fundamental de la estática de fluidos:

$$
d p=-\rho g d h
$$

donde la densidad $p$ es función de la altura $h$.

De la ecuación de los gases en función de la densidad se deduce: $\rho=\frac{p M}{R T}$

que sustituida en la anterior resulta:

$$
\frac{d p}{p}=-\frac{M g}{R T} d h
$$

expresión que combinada con la ecuación de Clapeyron dada anteriormente se obtiene:

$$
-\frac{d T}{d h}=\frac{M g}{c_{p}}
$$

es decir que se ha encontrado una expresión del gradiente adiabático de la atmósfera:

$$
\text { Grad }=\frac{M g}{c_{p}}
$$

Consultando tablas para la atmósfera terrestre a unos $23^{\circ} \mathrm{C}(300 \mathrm{~K})$ se encuentran los siguientes datos:

$$
c_{p}=29,10 \mathrm{Jmol}^{-1} \mathrm{~K}^{1} \quad M=28,90 \frac{\mathrm{g}}{\mathrm{mol}} \quad g=9,81 \frac{\mathrm{m}}{\mathrm{s}^{2}}
$$

Con lo que se obtiene para el gradiente adiabático de la atmósfera terrestre:

$$
\text { Grad }=-\frac{d T}{d h}=9,74 \frac{K}{k m}
$$

Así que para el caso del globo del ejemplo que se trata resultaría una disminución de temperatura de:

$$
1704 m=1,704 \mathrm{~km} \quad \Rightarrow \quad \Delta T=1,704 \mathrm{~km} \cdot\left(-9,74 \frac{\mathrm{K}}{\mathrm{km}}\right)=-16,6 \mathrm{~K}
$$


O sea que representa una disminución de temperatura de 16,6 grados de temperatura. Este valor de la variación de temperatura no puede considerarse determinante para influir de alguna forma en el cálculo propuesto.

\section{Algunas consideraciones finales}

Por las características del globo puede considerarse que el orden de magnitud del resultado es aceptable. Sin embargo se pueden discutir algunos aspectos:

- No se tiene en cuenta la posibilidad de que el globo explote debido a la disminución de presión exterior. Lo que habría que decir es que en todo momento el gas helio que hay en el interior del globo está en equilibrio de presión y temperatura con el aire exterior. La posibilidad de la explosión es muy poco probable para la relativamente poca altura que sube el globo. Además la experiencia demuestra que no es lo que suele ocurrir cuando un globo de este tipo se escapa cielo arriba.

Se puede determinar fácilmente la disminución de presión atmosférica a la altura que llega el globo. Volviendo a la expresión que relaciona el principio fundamental de la hidrostática con la ecuación de los gases ideales:

$$
\frac{d p}{p}=-\frac{M g}{R T} d h
$$

y pasando a integración (suponiendo que la temperatura influye poco, como se ha visto anteriormente, es decir es constante) resulta:

$$
\ln p=-\frac{M g h}{R T}+C
$$

Llamando $P_{0}$ a la presión en la superficie terrestre ( para $h=0$ ) entonces se cumple:

$$
\ln p_{0}=C \quad \text { y } \quad \ln \frac{p}{p_{0}}=-\frac{M g h}{R T}
$$

o bien:

$$
p=p_{0} e^{-\frac{M g h}{R T}}
$$

expresión llamada a veces ecuación barométrica la cual nos da la relación entre la presión y la altura.

Si sustituimos los parámetros del problema que estamos tratando, en la máxima altura $(1704 m)$ se tiene una presión de:

$$
p=1,013 \cdot 10^{5} \mathrm{~Pa} \cdot e^{-\frac{(0,0289 \mathrm{~kg} / \mathrm{mol}) \cdot\left(9,81 \mathrm{~m} / \mathrm{s}^{2}\right) \cdot(1704 \mathrm{~m})}{(8,31 \mathrm{~J} / \mathrm{mol} \mathrm{K}) \cdot(283,4 K)}}=8,25 \cdot 10^{4} \mathrm{~Pa}
$$

o lo que es lo mismo 0,81 atm. Se ha tomado la temperatura teniendo presente la disminución calculada anteriormente respecto a la temperatura de la superficie terrestre (considerada de $\left.23^{\circ} \mathrm{C}=300 \mathrm{~K}\right)$, es decir $300 K-16,6 K=283,4 K$.

Por tanto la disminución de presión respecto a la superficie $(0,81 \mathrm{~atm}-1$ atm $=-0,19 \mathrm{~atm}$ ) puede considerarse 
de poca entidad para perjudicar al globo en su ascenso.

- Otro punto a discutir sería la fricción del aire sobre el globo al ir subiendo. Evidentemente debido a dicha fricción habrá una disipación de calor y por tanto el proceso no sería adiabático. Se desprecia este efecto en el cálculo.

- El razonamiento se basa, como factor más importante, en el hecho que el globo dejará de subir cuando se iguala la fuerza ascensional con su peso.

- También se podría discutir qué es lo que sucederá cuando el globo alcance la máxima altura: ¿el globo se quedará allí ... o volverá a la Tierra...?. ¿Qué fenómenos influirán en el destino final del globo? Pero esto sería tema para otro artículo.

\section{Nota}

1 La ecuación de Clapeyron suele deducirse partiendo de querer averiguar lo que ocurre en un sistema en equilibrio cuando se modifican infinitamente poco las condiciones exteriores. Basta, para ello, expresar que, tanto el estado inicial como el final, son estados de equilibrio, con lo cual se cumple $\delta G=0$ (no hay variación de la entalpía libre). La forma usual de escribir la ecuación de Clapeyron es:

$$
-\frac{\delta Q}{T} d T+\delta V d p=0
$$

Expresión que con el apoyo de las relaciones:

$$
p \delta V=n R \delta T \quad \delta Q=n c_{p} \delta T
$$

nos conduce a:

$$
\frac{d T}{T}=\frac{R}{c_{p}} \frac{d p}{p}
$$

\section{Referencias bibliográficas}

Castro y Cañadas. Temas de Física. Madrid: Editorial Multicop. 1990.

Fernández J., Pujal M. Curso de Física General. Barcelona: Editorial Reverté. 1990.

Fornells y otros. Física. Barcelona: Editorial Teide. 1990.

Frish, Timoreva. Curso de Física general. Moscú: Editorial MIR. 1989.

Glasstone S. Termodinámica para quimicos. Barcelona: Editorial Aguilar. 1990.

Generalidad de Cataluña. Curricula Batxillerat. Barcelona: Departamento de Enseñanza. 1999.

Gray H.B., Haight G:P. Principios básicos de química. Barcelona: Editorial Reverté. 1980.

Pallisa y otros. 100 Problemas de termodinámica. Editorial Paraninfo. 2000.

Physical Science Study Committee. Física. Barcelona: Editorial Reverté. 1980.

Resnick-Halliday. Física General. México: Editorial CECSA. 1985.

Sears F.W. Termodinámica. Barcelona: Editorial Reverté. 1989.

Sears F.W. Mecánica, calor y sonido. Barcelona: Editorial Aguilar. 1991.

Tipler P. Física. Barcelona: Editorial Reverté. 2002. 